\title{
INFLUÊNCIA DAS CARACTERÍSTICAS SOCIODEMOGRÁFICAS E OCUPACIONAIS EM TRABALHADORES DA EQUIPE DE ENFERMAGEM COM UM EMPREGO E MULTIEMPREGO ${ }^{1}$
}

\author{
THE INFLUENCE OF SOCIO-DEMOGRAPHIC AND OCCUPATIONAL \\ CHARACTERISTICS IN NURSING TEAM WORKERS WITH A JOB AND \\ MORE THAN ONE JOB
}

\section{INFLUENCIA DE LAS CARACTERÍSTICAS SOCIODEMOGRÁFICAS Y OCUPACIONALES EN TRABAJADORES DEL EQUIPO DE ENFERMERÍA CON UN EMPLEO Y MULTIEMPLEO}

\author{
Ana Claudia Yassuko Murassaki ${ }^{*}$ \\ Willian Augusto de Melo ${ }^{* *}$ \\ Laura Misue Matsuda ${ }^{* *}$
}

\begin{abstract}
RESUMO
Estudo transversal, pré-experimental por comparação com grupo estático, que teve como objetivo verificar a associação de características sociodemográficas e ocupacionais em trabalhadores de enfermagem com um emprego e multiemprego. Aplicou-se um questionário, adaptado do "Inventário Sócio-Demográfico e Ocupacional" e "Indice de Capacidade para o Trabalho", a 208 trabalhadores de enfermagem com um emprego e 52 com multiemprego, que atuavam em um hospital universitário público. $\mathrm{Na}$ análise dos dados, realizaram-se o teste Exato de Fisher e teste Qui-Quadrado de Yates Corrigido, com intervalo de confiança de 95\% e nível de significância de 5\%. Constatou-se que existe associação significativa entre o número de empregos e algumas variáveis sociodemográficas e ocupacionais (sexo masculino; não realização de horas extras e afastamento por doença relacionada ao trabalho); o sexo masculino e a não realização de horas extras aumentam a razão de chance em 3,09 e 6,43, respectivamente, de ter multiemprego; e o afastamento por doença relacionada ao trabalho consiste em fator de proteção para não assumir mais de um emprego. Concluiu-se que a maioria das variáveis sociodemográficas e ocupacionais investigadas não exerce influência significativa nos trabalhadores com multiemprego, mas é necessário melhorar o aporte teórico acerca dessa condição e suas consequências para que, a partir disso, se estabeleçam estratégias que auxiliem no seu enfrentamento e promovam a saúde de seus trabalhadores.
\end{abstract}

Palavras chave: Carga de trabalho, trabalhadores, enfermagem, avaliação da capacidade de trabalho.

\footnotetext{
ABSTRACT

Cross-sectional study, pre-experimental by comparison with static group, which aimed to check the association of socio-demographic and occupational characteristics in nursing workers with one job and more than one job. A questionnaire adapted from "Socio-Demographic and Occupational Inventory" and "Work Ability Index", to

${ }^{1}$ Parte da Dissertação de Mestrado de título "Capacidade para o trabalho de trabalhadores de enfermagem com um e mais empregos".

*Enfermeira. Docente da Universidade Estadual de Maringá (UEM). Maringá, Paraná, Brasil. Email: anamurassaki@yahoo. com.br.

${ }^{* *}$ Enfermeiro. Docente do Centro Universitário de Maringá (Cesumar). Brasil. Email: profewill@yahoo.com.br.

*** Enfermeira. Docente do Departamento de Enfermagem da Universidade Estadual de Maringá (UEM). Maringá, Paraná, Brasil.Email:1mmatsuda@uem.br.
} 
208 nursing workers with a job and with 52 with more than one job, who worked in a public university hospital, was applied. For data analysis, the Fisher's Exact test and Yates' Chi-square test, were used with an interval of $95 \%$ and significance level of $5 \%$. It was noticed that there is a significant association between the number of jobs and some socio-demographic and occupational variables (male, non-completion of overtime and sick leave related to work), the male and non-completion of overtime increase the odds ratio at 3.09 and 6.43 , respectively, of having multi-role job; and the sick leave related to work turns out to be a protective factor for not taking more than one job. It was concluded that most socio-demographic and occupational study variables did not significantly influence in workers with more than one job, but it's necessary to improve the theoretical basis about this condition and its consequences so from this issue on, the strategies to assist in their achievement and promote the health of their workers can be established.

Key words: Workload, workers, nursing, work capacity evaluation.

\section{RESUMEN}

Estudio transversal, pre-experimental por comparación con el grupo estático, que tuvo como objetivo verificar la asociación de características sociodemográficas y laborales en trabajadores de enfermería con un empleo y multiempleo. Se aplicó un cuestionario adaptado del "Inventario sociodemográfico y ocupacional" e "Índice de capacidad de trabajo", a 208 trabajadores de enfermería con un empleo y con 52 trabajadores con multiempleo, que trabajaban en un hospital universitario público. En el análisis de datos se utilizó la Prueba exacta de Fisher y la Prueba de Chi-cuadrado de Yates corregida, con un intervalo de 95\% y un nivel de significación de 5\%. Se encontró que existe una asociación significativa entre el número de empleos y algunas variables sociodemográficas y ocupacionales (hombres, la no realización de las horas extras y alejamiento por enfermedad relacionada con el trabajo); el sexo masculino y la no realización de horas extras aumentan la odds ratio a 3,09 y 6,43, respectivamente, de tener multiempleo; y el alejamiento por enfermedad relacionada con el trabajo constituye en factor de protección para no asumir más de un empleo. Se concluyó que la mayoría de las variables sociodemográficas y laborales investigadas no influenciaron significativamente en los trabajadores con multiempleo, pero es necesario mejorar la base teórica acerca de la enfermedad y sus consecuencias para que, a partir de esto, se establezcan estrategias que ayuden en su enfrentamiento y promuevan la salud de los trabajadores.

Palabras clave: Carga de trabajo, trabajadores, enfermería, evaluación de capacidad de trabajo.

Fecha recepción: 23/11/11 Fecha aceptación: 18/03/13

\section{INTRODUÇÃO}

Na contemporaneidade, o sistema capitalista marcado pelo processo de globalização; aumento da competitividade; intensificação do ritmo laboral; busca por melhores condições de vida e; acumulação de bens, tem levado os indivíduos a trabalharem excessivamente (1). Apesar da importância do trabalho à vida do Homem e à sociedade, a busca pela produtividade descomedida tem ocasionado riscos à saúde, sofrimento e adoecimento do trabalhador (2).

Em se tratando de profissões da área da saúde, sobretudo da enfermagem, o risco de sobrecarga de trabalho e de contrair doenças é ainda maior porque, os seus trabalhadores, quase sempre atuam por longos períodos (3) e em contanto frequente com fatores que podem causar dano à sua saúde física e mental, tais como: longa jornada laboral; trabalho noturno; manipulação de produtos químicos; exposição à radiação ionizante; sustentação de excesso de peso durante a assistência ao paciente; dentre outros (4).

Além dos fatores citados, o processo de produção-trabalho da enfermagem é representado pelo trabalho manual, que tem evoluído pouco tecnologicamente, inserido em 
uma organização e divisão que visa à intensificação do ritmo de trabalho, ao aumento da produtividade, à prática parcelada e rotinizada, aliado ao trabalho em turnos, o qual facilita a conciliação e o exercício de duas ou mais escalas de trabalho (4).

Sabe-se que o excesso ou sobrecarga de trabalho, decorrente de duas ou mais jornadas, também denominado multiemprego, pode exigir muito do organismo do trabalhador e dificultar a sua recuperação, expondo-o, inclusive, ao risco de morte (1).

No que se refere ao absenteísmo por doença na enfermagem, à literatura aponta que os trabalhadores que têm multiemprego apresentam maior índice de absenteísmo-doença quando comparados àqueles com apenas um emprego (5) e esse fato, em parte, pode ser explicado pelo maior tempo de permanência do profissional no ambiente hospitalar e em contato com o paciente, expondo-o por mais tempo a cargas psíquicas, biológicas, químicas, físicas, mecânicas e fisiológicas (3).

Pesquisas indicam que os profissionais de enfermagem com multiemprego são mais propensos ao estresse, pois não descansam tempo suficiente entre as jornadas de trabalho e, associado ao pouco tempo para se dedicarem ao autocuidado e ao lazer, têm a sua qualidade de vida prejudicada $(1,5)$.

Ao ponderar que a enfermagem foi eleita pela Health Education Authority como a quarta profissão mais estressante (6), acredita-se que o aumento da jornada de trabalho decorrente do multiemprego pode acarretar riscos à saúde destes profissionais, os quais, não raro, apresentam sinais de sofrimento e até mesmo psicopatologias advindas do trabalho (1).

Cumpre salientar que a produção científica referente ao multiemprego entre profissionais de enfermagem necessita ser mais explorada, sobretudo no que diz respeito à capacidade para o trabalho daqueles que se encontram nessa condição. Perante esse fato, a presente pesquisa se justifica porque é importante e necessário melhorar o aporte de conhecimento acerca do tema, identificando-se os fatores associados ao multiemprego entre trabalhadores de enfermagem para, a partir disso, instituírem-se estratégias que auxiliem no enfrentamento ou minimização dos seus efeitos.

Como questão que direciona a presente investigação, estabeleceu-se: Os fatores sociodemográficos e ocupacionais influenciam no número de empregos em profissionais de enfermagem? Para responder a essa indagação, propõe-se a realização deste estudo, que tem como objetivo verificar a associação de características sociodemográficas e ocupacionais de trabalhadores de enfermagem com um emprego e com multiemprego.

\section{METODOLOGIA}

Estudo transversal, pré-experimental por comparação com grupo estático, realizado em um hospital universitário público do Norte do Paraná, nos meses de fevereiro a abril de 2011, com trabalhadores de enfermagem lotados na Diretoria de Enfermagem.

Para fins deste estudo, o Grupo 1, considerado grupo controle estático, é formado por profissionais de enfermagem com um emprego e o Grupo 2 é aquele composto por trabalhadores que mantêm multiemprego.

Para a composição da amostra, primeiramente, realizaram-se visitas em todas as unidades, nos três turnos de trabalho e indagou-se aos enfermeiros supervisores quantos funcionários sob sua supervisão mantinham multiemprego. O resultado foi 141 trabalhadores de enfermagem com multiemprego e 542 com apenas um emprego.

De posse desses dados, calculou-se uma amostra estratificada proporcional, na qual foi respeitada a proporcionalidade de cada categoria profissional - Enfermeiro (Enf); Técnico de Enfermagem (TE); Auxiliar de Enfermagem (AE) e Atendente de Enfermagem (At E), em ambos os grupos. 
O Grupo 1 se constituiu com 236 profissionais, distribuídos da seguinte maneira: 17 At E; 120 AE; 59 TE e 40 Enf e o Grupo 2, com 59 trabalhadores: 01 At E; 29 AE; 19 TE e 10 Enf. A somatória de sujeitos nos dois Grupos perfizeram um total de 295 trabalhadores, com margem de segurança de 20\% devido a eventuais perdas.

Vale destacar a presença da categoria Atendente de Enfermagem (At E) que, de acordo com a Lei Federal 7498/1986 (7), deveria ser extinta em dez anos, a partir da data da sua promulgação. No entanto, a Lei Federal 8967/1994 (8) alterou a redação da lei anterior e assegurou o direito do exercício da Enfermagem a quem tivesse sido admitido antes da vigência da lei 7498/1986 (7).

Ressalta-se que a legislação brasileira (8) permite que os Atendentes de Enfermagem realizem atividades elementares relacionadas à promoção da assistência de enfermagem eficiente, mas não deve envolver o cuidado direto ao paciente.

Para a coleta de dados, utilizou-se um instrumento constituído de duas Partes: a Parte I foi adaptada a partir do "Inventário Sócio-Demográfico e Ocupacional” elaborado por Domansky² é composta por 20 questões estruturadas e semiestruturadas, as quais buscam informações relacionadas ao perfil sociodemográfico e ocupacional dos sujeitos. Já a Parte II do instrumento, o "Índice de Capacidade para o Trabalho” (ICT), é composto por dez questões fechadas que avaliam a capacidade para o trabalho, a partir da percepção do próprio trabalhador, sendo constituída por um questionário autoaplicável, elaborado e validado por pesquisadores finlandeses (9).

A saber, o ICT é composto por questões relativas às exigências físicas e mentais do trabalho, ao estado de saúde, bem como aos recursos individuais do trabalhador para o enfrentamento do trabalho (9) e no Brasil, esse instrumento foi traduzido por um grupo de pesquisadores liderados pela Dra. Frida Marina Fischer (9) e recentemente, teve testado a sua validade de construto e de confiabilidade, em profissionais de enfermagem (10).

No ICT, o escore pode variar de 7 a 49 pontos e a capacidade para o trabalho é classificada em Adequada e Inadequada, utilizando-se o limite de referência de 41, para trabalhadores com idade igual ou inferior a 35 anos e, 37, para maiores de 35 anos.

No tratamento dos dados, utilizou-se o programa Microsoft Office Excel $2007^{\circledR}$ e, na análise estatística descritiva e inferencial, o programa StatSoft Statística $8.0^{\varpi}$. Considerou-se como variável dependente o número de empregos e como variáveis independentes, o perfil sociodemográfico, ocupacional e o ICT propriamente dito. Além disso, na análise inferencial dos dados, aplicou-se o Teste Exato de Fischer para as variáveis binomiais e o Teste Qui-Quadrado de Yates Corrigido para as demais variáveis, considerando o intervalo de confiança de 95\% e nível de significância de 5\%, em ambos os testes.

Cabe mencionar que, para a realização desta pesquisa, todas as exigências estabelecidas na Resolução 196/1996 (11) foram respeitadas e o Parecer favorável foi emitido pelo Comitê de Ética em Pesquisas Envolvendo Seres Humanos (CEP), da Universidade Estadual de Londrina (UEL), sob o Parecer $n^{0} 253 / 2010$.

\section{RESULTADOS}

Dentre os 295 trabalhadores investigados, 6 encontravam-se de licença-médica; 3 de licença-maternidade ou adoção; 6 de licençaprêmio; 03 de férias ou licença-coletiva; 1 
havia solicitado demissão e 16 se recusaram a participar, totalizando 35 perdas. Os demais 260 trabalhadores aceitaram participar da pesquisa.

Na Tabela 1 constam as informações referentes às variáveis sociodemográficas dos su- jeitos, bem como aquelas que se associaram ao número de empregos.

No tocante aos resultados das variáveis ocupacionais e a classificação da capacidade para o trabalho, observa-se na Tabela 2 associação significativa entre o número de

Tabela 1. Distribuição das variáveis sociodemográficas, de acordo com o número de emprego dos profissionais de enfermagem $(\mathrm{n}=260)$, de um hospital universitário público do Norte do Paraná. Brasil, 2011.

\begin{tabular}{|c|c|c|c|c|c|c|c|}
\hline \multirow[b]{3}{*}{ VARIÁVEIS } & \multicolumn{4}{|c|}{ NÚMERO DE EMPREGO } & \multirow{2}{*}{\multicolumn{3}{|c|}{ Análise Estatística }} \\
\hline & \multicolumn{2}{|c|}{ Multiemprego } & \multicolumn{2}{|c|}{ Um Emprego } & & & \\
\hline & n & $\%$ & $\mathbf{n}$ & $\%$ & $\mathrm{OR} \ddagger$ & $\mathrm{IC}+95 \%$ & p-valor \\
\hline Sexo & & & & & 3,09 & $(1,51-6,34)$ & $0,0011^{\star}$ \\
\hline Masculino & 20 & 7,69 & 35 & 13,46 & & & \\
\hline Feminino & 32 & 12,31 & 173 & 66,54 & & & \\
\hline Faixa etária & & & & & 0,83 & $(0,33-2,14)$ & $0,6540^{*}$ \\
\hline$\leq 35$ anos & 8 & 3,1 & 27 & 10,47 & & & \\
\hline$>35$ anos & 44 & 17,05 & 179 & 69,38 & & & \\
\hline Estado Civil & & & & & 0,87 & $(0,43-1,77)$ & $0,7424^{\star}$ \\
\hline Casado & 35 & 13,89 & 132 & 52,38 & & & \\
\hline Não casado & 16 & 6,35 & 69 & 27,38 & & & \\
\hline Escolaridade & & & & & 1,16 & $(0,60-2,22)$ & $0,6453^{\star}$ \\
\hline Superior & 25 & 9,73 & 106 & 41,25 & & & \\
\hline Não superior & 27 & 10,51 & 99 & 38,52 & & & \\
\hline Renda Familiar & & & & & 1,4 & $(0,71-2,77)$ & $0,3351^{\star}$ \\
\hline$>\mathrm{R} \$ 3600^{\#}$ & 27 & 11,25 & 92 & 38,33 & & & \\
\hline$\leq \mathrm{R} \$ 3600^{\#}$ & 21 & 8,75 & 100 & 41,67 & & & \\
\hline Dependentes & & & & & 1,54 & $(0,64-3,82)$ & $0,3430^{*}$ \\
\hline Não & 8 & 3,1 & 45 & 17,44 & & & \\
\hline Sim & 44 & 17,05 & 161 & 62,4 & & & \\
\hline
\end{tabular}

* Nível descritivo para o Teste Exato de Fisher ( $p$-valor significativo $\leq$ 0,05).

$\dagger$ IC - Intervalo de Confiança 95\%.

‡ OR - Odds Ratio (Razão de Chance)

* US\$1,00 (Dólar) = R\$2,29 (Reais)

Tabela 2. Distribuição das variáveis ocupacionais, de acordo com o número de emprego dos profissionais de enfermagem ( $\mathrm{n}=260$ ), de um hospital universitário público do Norte do Paraná. Brasil, 2011.

\begin{tabular}{|c|c|c|c|c|c|c|c|}
\hline \multirow[b]{3}{*}{ VARIÁVEIS } & \multicolumn{4}{|c|}{ NÚMERO DE EMPREGO } & \multirow{2}{*}{\multicolumn{3}{|c|}{ Análise Estatística }} \\
\hline & \multirow{2}{*}{\multicolumn{2}{|c|}{ Multiemprego }} & \multicolumn{2}{|c|}{ Um Emprego } & & & \\
\hline & $\mathbf{n}$ & & $\mathbf{n}$ & $\%$ & $\mathrm{OR} \ddagger$ & $\mathrm{IC} \dagger 95 \%$ & p-valor \\
\hline \multicolumn{8}{|l|}{ Ocupação } \\
\hline Atendente & 1 & 0,38 & 14 & 5,38 & 0,27 & $(0,01-2,05)$ & $0,3170^{* *}$ \\
\hline Auxiliar & 24 & 9,23 & 106 & 40,77 & 0,82 & $(0,43-1,58)$ & $0,6418^{* *}$ \\
\hline Técnico & 17 & 6,54 & 53 & 20,38 & 1,42 & $(0,70-2,88)$ & $0,3821^{* *}$ \\
\hline Enfermeiro & 10 & 3,85 & 35 & 13,46 & 1,18 & $(0,50-2,72)$ & $0,8376^{* *}$ \\
\hline
\end{tabular}


Continuação Tabela 2.

\begin{tabular}{|c|c|c|c|c|c|c|c|}
\hline Tempo na instituição & & & & & 1,82 & $(0,92-3,59)$ & $0,0644^{*}$ \\
\hline$\leq 16$ anos & 34 & 13,08 & 106 & 40,77 & & & \\
\hline$>16$ anos & 18 & 6,92 & 102 & 39,23 & & & \\
\hline \multicolumn{8}{|l|}{ Turno } \\
\hline Diurno & 36 & 13,85 & 119 & 45,77 & 1,68 & $(0,84-3,40)$ & $0,1550^{* *}$ \\
\hline Noturno & 11 & 4,23 & 69 & 26,54 & 0,54 & $(0,24-1,17)$ & $0,1306^{* *}$ \\
\hline Revezamento & 5 & 1,92 & 20 & 7,69 & 1 & $(0,31-3,02)$ & $0,7925^{\star *}$ \\
\hline Horas extras & & & & & 6,43 & $(3,11-13,35)$ & $0,0000^{\star}$ \\
\hline Não & 26 & 10 & 28 & 10,77 & & & \\
\hline Sim & 26 & 10 & 180 & 69,23 & & & \\
\hline Estuda & & & & & 1,11 & $(0,56-2,21)$ & $0,8722^{*}$ \\
\hline Não & 34 & 13,08 & 131 & 50,38 & & & \\
\hline Sim & 18 & 6,92 & 77 & 29,62 & & & \\
\hline Problema de saúde relacionado ao trabalho & & & & & 1,27 & $(0,62-2,63)$ & $0,5103^{*}$ \\
\hline Não & 15 & 5,88 & 74 & 29,02 & & & \\
\hline Sim & 34 & 13,33 & 132 & 51,76 & & & \\
\hline Afastamento por doença relacionada ao trabalho & & & & & 0,39 & $(0,16-0,92)$ & $0,0246^{*}$ \\
\hline Não & 44 & 16,92 & 142 & 54,62 & & & \\
\hline $\operatorname{Sim}$ & 8 & 3,08 & 66 & 25,38 & & & \\
\hline Acidente de trabalho & & & & & 0,92 & $(0,48-1,78)$ & $0,8763^{*}$ \\
\hline Não & 30 & 11,54 & 116 & 44,62 & & & \\
\hline $\operatorname{Sim}$ & 22 & 8,46 & 92 & 35,38 & & & \\
\hline Capacidade de trabalho & & & & & 0,47 & $(0,18-1,16)$ & $0,0953^{*}$ \\
\hline Adequada & 39 & 15 & 120 & 46,15 & & & \\
\hline Inadequada & 13 & 5 & 88 & 33,85 & & & \\
\hline
\end{tabular}

* Nível descritivo para o Teste Exato de Fisher ( $p$-valor significativo $\leq 0,05$ )

** Nível descritivo para o Qui-quadrado de Yates Corrigido

$\dagger$ IC - Intervalo de Confiança 95\%.

‡ OR - Odds Ratio (Razão de Chance)

empregos com a "realização de horas extras", bem como "afastamento por doença relacionada ao trabalho".

\section{DISCUSSÃO E CONCLUSÃO}

Ao examinar as características sociodemográficas dispostas na Tabela 1, observa-se que o sexo foi à única variável a qual se associou significativamente ao número de empregos. Segundo dados da referida Tabela, embora o número de mulheres com multiemprego (32 $=12,31 \%$ ) seja maior do que o de homens $(20=7,69 \%)$, proporcionalmente houve predominância masculina nessa condição.

Ao considerar que a sociedade traz ar- raigada a visão tradicional de que o homem tem a responsabilidade de ser o provedor da família, não surpreende o fato de se ter obtido uma razão de chance para o multiemprego de 3,09 vezes maior em homens do que em mulheres. Apesar de ser evidente a transformação na composição do domicílio, revelada pelo crescimento relativo de famílias chefiadas por mulheres nos últimos anos, o modelo patriarcal, como mostra este estudo, ainda não foi superado (12).

Ressalta-se que o trabalho doméstico tem papel preponderante na vida das mulheres trabalhadoras, as quais são maioria na enfermagem, pois o lugar delas permanece atribuído ao lar, à maternidade e ao do cuidar dos outros (13). Os múltiplos papéis assumidos pelas mulheres trabalhadoras tendem a gerar 
situações de conflito e estresse, ainda mais quando a trabalhadora mantém multiemprego, visto que a sua inserção no mercado de trabalho não a desvinculou dos afazeres domésticos e da educação dos filhos, resultando em acúmulo de atribuições $(1,13)$.

Vale destacar que dentre os 52 trabalhadores que mantinham multiemprego, 44 $(84,6 \%)$ possuíam mais de 35 anos. Esse dado pode se pautar no fato de que, geralmente, pessoas nessa faixa etária já constituíram família e, por sofrerem com problemas de baixa remuneração, muitas vezes, necessitam de outro(s) emprego(s) para complementar a sua renda (1).

$\mathrm{O}$ achado antes mencionado merece atenção porque a enfermagem, por si só, é uma profissão desgastante dada à sobrecarga de trabalho, a qual se acentua com a manutenção do multiemprego (14). Aliado a isso, um estudo finlandês apontou que a capacidade para o trabalho tende a reduzir com o passar da idade e, a partir dos 35 anos, a capacidade para o trabalho tende a declinar-se (15).

A Tabela 1 evidencia também que dentre o grupo de trabalhadores com multiemprego, a maioria $(35=13,89 \%)$ era casado e possuía dependentes $(44=17,05 \%)$. Esses resultados corroboram com os dados de um estudo realizado em um hospital de oncologia pediátrica (16), o qual apontou que os mais jovens e solteiros pertenciam ao grupo que exerciam apenas um emprego.

Diante das maiores responsabilidades afetiva e financeira, demandadas pela família, o profissional que detém multiemprego tende à maior satisfação por estar menos preocupado com suas finanças e ser mais reconhecido pelos seus colegas de trabalho, em detrimento dos momentos de lazer. Isso possivelmente ocorre porque o modelo capitalista pode induzir o trabalhador ao escravismo do consumo, imprimindo a ideia de que o homem digno é aquele que trabalha muito, sobretudo se for o responsável pela subsistência da família (17).

A ênfase na vida profissional em detri- mento da vida privada, revelada pela extensa carga horária advinda dos múltiplos empregos, tem reduzido o tempo de lazer, de convívio social e de integração familiar entre esses profissionais (1). Essa condição necessita ser melhorada porque, a sobrecarga de trabalho e o estresse, tendem a resultar em prejuízos à saúde do profissional e ao seu trabalho (14).

De acordo com a Tabela 2, as variáveis ocupacionais que se associaram significativamente com o número de empregos foram: "realização de horas extras"; e "afastamento por doença relacionada ao trabalho".

A variável "realização de horas extras" indica que os profissionais que não faz horas extras possuem razão de chance para manutenção de multiemprego 6,43 vezes maior do que aquele que faz hora extra. Isso, possivelmente, deve-se ao fato de que aqueles os quais acumulam jornadas de trabalho têm menos tempo para assumir responsabilidades após o término do expediente de trabalho.

A literatura aponta o multiemprego como fonte de estresse físico e emocional (18) que pode fragilizar a saúde dos trabalhadores nessa condição. Desse modo, a opção de não fazer hora extra, pode ser uma medida de defesa adotada pelos profissionais com multiemprego, para não se exporem aos riscos e às condições de trabalho desfavoráveis além do tempo normal de trabalho.

Destaca-se, ainda, que os profissionais de enfermagem os quais mantêm outros empregos, geralmente o faz em busca de melhores salários (19), e isso pode ser um provável motivo que explica o fato de os trabalhadores nessa condição não realizarem horas extras.

A segunda variável ocupacional (Tabela 2) associada ao multiemprego foi o "afastamento por doença relacionada ao trabalho", apontada como fator de proteção para a manutenção de multiemprego, ou seja, os profissionais que apresentaram afastamento por doença relacionada ao trabalho possuem menor razão de chance $(0,39)$ de manter multiemprego se comparados àqueles que não relataram afastamento. 
A relação antes mencionada pode ter ocorrido devido ao corte transversal utilizado neste estudo, que permite apenas uma avaliação pontual de determinado evento e, por isso, deve-se considerar a limitação do "efeito do trabalhador sadio" que corresponde ao risco de subestimar a ocorrência dos problemas de saúde, uma vez que os trabalhadores em atividade seriam aqueles mais saudáveis e aptos para o labor (20).

Os dados referentes à variável "afastamento por doença relacionada ao trabalho", obtidos neste estudo, contrastam com a literatura alusiva, a qual aponta que os profissionais com multiemprego estão mais propensos a apresentar absenteísmo-doença, se comparados àqueles com apenas um emprego (5).

No que se refere ao tempo de serviço na instituição, embora os testes estatísticos não tenham apresentado significância, percebese (Tabela 2) que dentre os 52 trabalhadores com multiemprego, 34 (13,08\%) apresentaram menor tempo de serviço ( $\leq 16$ anos), enquanto $18(6,92 \%)$ trabalhavam há mais tempo na instituição. Esse resultado pode ser decorrência do envelhecimento natural da idade, condição diminuidora da tolerância às altas cargas de trabalho, associada ao surgimento de doenças crônicas comuns nessa fase da vida (21).

No âmbito dos problemas de saúde relacionados ao trabalho, no grupo de trabalhadores com multiemprego, 15 (5,88\%) negaram apresentar qualquer tipo de problema, enquanto $34(13,33 \%)$ admitiram ter algum acometimento. O presente estudo não apontou diferença significativa entre os grupos com um emprego e multiemprego, e uma possível justificativa para esse achado pode estar pautada no fato de que neste estudo, foram incluídos somente os trabalhadores "sadios" ou que estavam trabalhando, excluindo-se aqueles em licença-médica.

É importante destacar que o trabalho pode ser prazeroso e mediador da saúde, desde que haja equilíbrio entre a carga psíquica causada pelo trabalho e a descarga dela (22). Nessa perspectiva, o trabalho nem sempre é patogênico, pois pode estabelecer o equilíbrio da saúde física e/ou mental do trabalhador e, ainda, fortalecer a sua identidade pessoal por manter contato com meio produtivo e também, com os outros que fazem parte do seu mundo de socializações (23).

Dentre os profissionais acometidos, ao analisar os problemas de saúde relacionados ao trabalho, percebeu-se que não há diferença entre os grupos, pois tanto o grupo de trabalhadores com multiemprego como o grupo com apenas um emprego apontou os acometimentos no sistema músculo-esquelético como sendo os mais frequentes, seguidos dos transtornos mentais e problemas respiratórios. Esse dado corrobora com os resultados de um estudo realizado com 528 trabalhadores de enfermagem de um hospital universitário do Rio Grande do Sul, o qual aponta que o ambiente hospitalar impõe condições desgastantes com conseqüências danosas à saúde osteomuscular desses profissionais (24).

Outro fator importante observado na Tabela 2, é a capacidade para o trabalho dos profissionais com multiemprego, pois consta que 39 (15\%) trabalhadores nessa condição apresentaram capacidade adequada e apenas $13(5 \%)$ apontaram capacidade inadequada. Esses dados não condizem com a literatura alusiva, a qual evidencia maior proporção de boa capacidade para o trabalho em trabalhadores que possuem apenas um emprego (25).

O fato de a maioria dos profissionais com multiemprego, investigados neste estudo, apresentar adequada capacidade para o trabalho pode ser explicada porque ela (a capacidade para o trabalho) é um construto multidimensional constituído pela associação entre recursos humanos individuais (saúde, capacidade funcional, experiência 
profissional, valores e atitudes) em relação às demandas do trabalho (ambiente, condições de trabalho, organização e supervisão) (15). Além disso, o fenômeno "efeito do trabalhador sadio", mencionado anteriormente, também deve ser considerado.

Verificou-se que houve associação estatisticamente significativa entre o número de empregos dos trabalhadores de enfermagem com a variável sociodemográfica "sexo"; com as variáveis ocupacionais "realização de horas extras"; e "afastamento por doença relacionada ao trabalho". Ainda com relação à variável sociodemográfica, evidenciou-se que o sexo masculino possui maior razão de chance para ter multiemprego, o que era esperado, já que o homem ainda é visto socialmente como o provedor da família.

Quanto à realização de horas extras, os dados apontaram que a não realização destas também consiste em maior razão de chance para a condição de multiemprego, podendo esse dado ser explicado, pelo menos em parte, pelo fato de que aqueles que possuem mais de uma jornada de trabalho têm pouco tempo disponível para trabalhar após o término do expediente normal.

No que se refere ao afastamento por doença relacionada ao trabalho, observou-se que os trabalhadores com afastamento anterior possuem menor razão de chance para a manutenção de multiemprego. A associação antes mencionada contrasta com a literatura alusiva, e pode ter ocorrido devido ao "efeito do trabalhador sadio", limitação comum em estudos de corte transversais.

Apesar de se ter encontrado poucas associações significativas entre o número de emprego e as variáveis sociodemográficas e ocupacionais, conclui-se que é preciso melhorar o aporte teórico acerca do multiemprego e suas consequências na enfermagem, por meio de estudos longitudinais para que, a partir disso, se estabeleçam estratégias que auxiliem no enfrentamento dessa condição e promovam a saúde de seus trabalhadores.

\section{REFERÊNCIAS}

1. Robazzi MLdoCC, Mauro MYC, Dalri RdeCdeMB, Silva LAda, Secco IAdeO, Pedrão LJ. Exceso de trabajo y agravios mentales a los trabajadores de la salud. Rev Cubana Enferm. 2010; 26(1): 52-64.

2. Fernandes SMBA, Medeiros SM, Ribeiro LM. Estresse ocupacional e o mundo do trabalho atual: repercussões na vida cotidiana das enfermeiras. REE-Bra- [Internet]. 2008 [citado 20 nov 2011]; 10(2): 414-27. Disponível em: http://www.fen. ufg.br/revista/v10/n2/v10n2a13.htm

3. Umann J, Guido LdeA, Freitas EdeO. Produção de conhecimento sobre saúde e doença na equipe de enfermagem na assistência hospitalar. Ciênc. cuid. saúde. 2011; 10(1): 162-8.

4. Manetti ML, Marziale MHP, Robazzi MLdoCC. Revisando os fatores psicossociais do trabalho de enfermagem. Rev Rene. 2008; 9(1): 111-9.

5. Costa FM, Vieira MA, Sena RR. Absenteísmo relacionado às doenças entre membros da equipe de enfermagem de um hospital escola. Rev. bras. enferm. 2009; 62(1): 38-44.

6. Teixeira RC, Mantovani MF. Enfermeiros com doença crônica: as relações com o adoecimento, a prevenção e o processo de trabalho. Rev. Esc. Enferm. USP. 2009; 43(2): 415-21.

7. Dispõe sobre a regulamentação do exercício da enfermagem e dá outras providências Brasil. Lei 7498/25 de junho de 1986. Diário Oficial da União, Brasília (DF), Seção 1, v.119, n.124, p.9273-5, (26-06-1986).

8. Altera a redação do art. 23 da lei 7498/86. Lei 8967/28 de dezembro de 1994. Diário Oficial da União, Brasília (DF), Seção 1, n.247, p.20829, (29-12-1994).

9. Tuomi K, Ilmarinen J, Jahkola A, Katajarinne L, Tulkki A. Índice de capacidade 
para o trabalho. São Carlos (SP): EdUFSCar; 2005. 59p.

10. Silva Junior SHA. Avaliação de qualidades psicométricas da versão brasileira do Índice de Capacidade para o Trabalho. 2010. [Dissertação mestrado]. [Rio de Janeiro]: Escola Nacional de Saúde Pública, Fundação Oswaldo Cruz; 2010. 99p.

11. Diretrizes e Normas Regulamentadoras de Pesquisas Envolvendo Seres Humanos. Resolução no 196/96/16 de outubro de 1996. Ministério da Saúde, Conselho Nacional de Saúde, (1996).

12. Lacerda ALR. Em busca dos indicadores biossociais da hipogamia. Estud Psicol. 2011; 15(1): 111-8.

13. Guerrer FJL, Bianchi ERF. Caracterização do estresse nos enfermeiros de unidades de terapia intensiva. Rev. Esc. Enferm. USP. 2008; 42(2): 355-62.

14. Schmoeller R, Trindade LL, Neis MB, Gelbcke FL, Pires DEP. Cargas de trabalho e condições de trabalho da enfermagem: revisão integrativa. Rev. gaúch. enferm. 2011; 32(2): 368-77.

15. Gould R, Ilmarinen J, Järvisalo J, Koskinen S. Dimensions of Work Ability: Results of the Health 2000 Survey. Helsinki, Finlandia: Waasa Graphics Oy; 2008.

16. Pafaro RC, Martino MMF. Estudo do estresse do enfermeiro com dupla jornada de trabalho em um hospital de oncologia pediátrica de Campinas. Rev. Esc. Enferm. USP. 2004; 38(2): 152-60.

17. Sentone ADD, Gonçalves AAF. Sofrimento no trabalho: significado para o auxiliar de enfermagem com dois vínculos empregatícios. Semina: Ciências Biológicas e da Saúde. 2002; 23(1): 33-8.

18. Oliveira LCB, Chaves-Maia EM. Saúde Psíquica dos Profissionais de Saúde em Hospitais Públicos. Rev. salud pública. 2008; 10(3): 405-13.

19. Dalri RdeCdeMB, Robazzi MLdoCC, Silva LAda. Riscos ocupacionais e alterações de saúde entre trabalhadores de enfermagem brasileiros de unidades de urgência e emergência. Cienc. enferm. 2010; 16(2): 69-81.

20. Martinez MC, Latorre MRDO. Saúde e capacidade para o trabalho de eletricitários do Estado de São Paulo. Ciênc. saúde coletiva. 2008; 13(3): 1061-73.

21. Inoue KC, Matsuda LM, Silva DMPP, Uchimura TT, Mathias TAdeF. Absenteísmo-doença da equipe de enfermagem em unidade de terapia intensiva. Rev. bras. enferm. 2008; 61(2): 209-14.

22. Dejours C, Abdoucheli E, Jayet C. Betiol MIS (coord). Psicodinâmica do trabalho: contribuições da Escola Dejouriana à análise da relação prazer, sofrimento e trabalho. São Paulo: Atlas; 1994. 145p.

23. Martins JT, Robazzi MLCC, Garanhani ML. Sentimentos de prazer entre enfermeiros de unidades de terapia intensiva. Cienc. enferm. 2009; 15(3): 45-53.

24. Magnago TSBS, Lisboa MTL, Griep RH, Kirchhof ALC, Guido LA. Aspectos psicossociais do trabalho e distúrbio musculoesquelético em trabalhadores de enfermagem. Rev. Latino-Am. Enfermagem. 2010; 18(3): 429-35.

25. Raffone AM, Hennington EA. Avaliação da capacidade funcional. 